\title{
Heterogeneity in neoadjuvant therapy for localized pancreatic cancer: a persisting radiant autonomy
}

\author{
Kjetil Søreide ${ }^{1,2,3}$ \\ ${ }^{1}$ Department of Gastrointestinal Surgery, Stavanger University Hospital, Stavanger, Norway; ${ }^{2}$ Department of Clinical Medicine, University of \\ Bergen, Bergen, Norway; ${ }^{3}$ Gastrointestinal Translation Research Unit, Stavanger University Hospital, Stavanger, Norway \\ Correspondence to: Prof. Kjetil Søreide, MD, PhD, FRCS(Edin) FACS. Department of Gastrointestinal Surgery, P.O. Box 8100, N-4068 Stavanger, \\ Stavanger University Hospital, Stavanger, Norway. Email: ksoreide@mac.com. \\ Comment on: Kizy S, Altman AM, Wirth KM, et al. Systemic therapy without radiation may be appropriate as neoadjuvant therapy for localized \\ pancreas cancer. Hepatobiliary Surg Nutr 2020;9:296-303.
}

Submitted Dec 05, 2020. Accepted for publication Jan 05, 2021.

doi: 10.21037/hbsn-2020-9

View this article at: http://dx.doi.org/10.21037/hbsn-2020-9

Pancreatic cancer remains a disease with an overall grim prognosis, even in the limited number of patients who are amenable for resection attempted at cure. Indeed, pancreatic cancer demonstrate a predisposition for invasive growth and distant metastasis-even in the very early stages of the disease. Alas, while surgical resection is the strongest factor for long-term survival, early recurrence after surgery is associated with a poor prognosis (1). In light of this knowledge and, despite the lack of good level I data, it seems that patients and practitioners have been voting with their feet regarding systemic therapy; an increasing number of institutions are giving systemic treatment before surgery with the hopes of better disease control and avoiding futile surgery. However, the contemporary practice is based on an "radiant autonomy" - as it would seem, that pretty much any regimen and combination of drugs and modalities will be offered in current practice, with the lack of randomized studies for most options but often with an institutional signature to preference and delivery.

With this backdrop in mind, it is of interest to read the current report from the University of Minnesota in this Journal (2). The investigators reviewed their practice over a 5-year period (2012 to 2016) for all patients deemed to have resectable pancreatic cancer (based on pre-treatment imaging studies) and who were given neoadjuvant chemotherapy prior to restaging for surgery. In their intention-to-treat evaluation, 25 (69\%) proceeded to have surgical resection out of the 36 patients who had neoadjuvant therapy. Among these 25,3 had portal vein resection, 20 had a pancreatoduodenectomy and 5 a distal resection. Among the 36 patients, there were no local progression of disease during neoadjuvant therapy, and 'inoperability' came from development of distant metastasis or declaring of underlying comorbidity and failure to tolerate the treatment. While the favorable survival times and resection rates are obviously important and welcomed results for the individual patients, the comparison of the smaller groups are not powered for generalizability or to confirm associations. The investigators have clearly and deliberately taken on an upfront systemic therapy strategy for resectable pancreatic cancer in their institution and, should be congratulated for presenting their data so that others may learn from their experience while we await results from ongoing randomized trials. While all patients were discussed in a tumor board, recent data have shown just how diverse decisions can be (3).

Perhaps the most important notion from this report may be their omission of radiotherapy as part of upfront systemic disease control, as this have been proposed by several institutions across the United States. However, as the investigators noted (2), no patients dropped out from surgical resection due to local disease progression. Rather, distant metastasis and patients who declared themselves as unfit due to complications and poor tolerability were the main reasons for not proceeding to surgery. So, would systemic chemotherapy suffice in the neoadjuvant setting? Is there any added benefit to radiotherapy that justifies the added toxicity? Currently, there seems to be 
'perfect autonomy' with arguments on either side for and against for either approach, particularly when considering chemoradiotherapy (4).

Available data based on randomized trials that investigate systemic therapy in a neoadjuvant setting for resectable pancreatic cancer each have their own flaws when trying to address and compare the data. For example, in the PACT-15 trial (5), designed as a 3-armed phase II/III study, only one of the three arms received systemic chemotherapy (Gemcitabine-based multidrug regimen). While the neoadjuvant arm fared best in the PACT-15 trial, the sample was small and, the trial preceded the use of more potent multidrug regimens currently preferred. In a Japanese phase II/III trial, the multidrug regimen is a combination of Gemcitabine with S-1 (6), a drug that is preferably used in Japan, but rarely used outside this region. In the University of Minnesota cohort (2), gemcitabine was the most frequently used drug, either as single agent $(\mathrm{n}=17 ; 47 \%)$ or multidrug regimen $(\mathrm{n}=8 ; 22 \%)$ with 11 (31\%) starting on FOLFIRINOX, probably as this drug combination popularized first for metastatic cancer (7) and only later with superior results compared to gemcitabine in the adjuvant setting (8). The recent PREOPANC-1 trial from the Dutch Pancreatic Cancer Group also investigated Gemcitabine-based regimens (9) with a short-course chemoradiotherapy in the experimental arm, and reported no statistical difference achieved for the primary endpoint of overall survival between the two groups. Notably, the PREOPANC trial included both resectable and borderline tumors of all locations in the pancreatic gland, and only superior outcomes where found for the neoadjuvant strategy in the borderline tumours.

Currently, use of FOLFIRINOX is preferred as the standard when tolerated and seems to have the most promising efficacy. Thus, one can rightfully ask if radiotherapy is needed in addition in the neoadjuvant setting. Centers have reported increased adverse events, particularly in relation to tumors abutting the vessels. Others have reported a more favorable oncological outcome in exactly those who are considered borderline resectable $(4,10)$, adding layers of confusion and shaded areas to the ongoing debate. Effective systemic chemotherapy may be more important than the added radiation (11), although the true effect of either has yet to be declared. Until more robust data eventually emerge, preferably based on solid phase III studies, there is autonomy in contemporary practice in the use of neoadjuvant therapy. This multifaceted question is in in need for a more collective solution than what currently exists as radiant autonomy. As we learn more about the effect of multidrug regimens and their effects, and see the emergence of personalized drugs and immunotherapy, we should focus on the actual role and contribution to oncological control provided by radiotherapy in pancreatic cancer. It is perceived that this can only come from welldesigned and adequately powered trials that address this question. Thus, we await these trials eagerly to possibly inform the best clinical practice and in order to provide informed decisions to patients with otherwise resectable, localized pancreatic cancer. For now, the jury is still out.

\section{Acknowledgments}

Funding: None.

\section{Footnote}

Provenance and Peer Review: This article was commissioned by the editorial office of Hepatobiliary Surgery and Nutrition. The article did not undergo external peer review.

Conflicts of Interest: The author has completed the ICMJE uniform disclosure form (available at https://hbsn. amegroups.com/article/view/10.21037/hbsn-2020-9/coif). The author has no conflicts of interest to declare.

Ethical Statement: The author is accountable for all aspects of the work in ensuring that questions related to the accuracy or integrity of any part of the work are appropriately investigated and resolved.

Open Access Statement: This is an Open Access article distributed in accordance with the Creative Commons Attribution-NonCommercial-NoDerivs 4.0 International License (CC BY-NC-ND 4.0), which permits the noncommercial replication and distribution of the article with the strict proviso that no changes or edits are made and the original work is properly cited (including links to both the formal publication through the relevant DOI and the license). See: https://creativecommons.org/licenses/by-nc-nd/4.0/.

\section{References}

1. Tanaka M, Mihaljevic AL, Probst P, et al. Meta-analysis of recurrence pattern after resection for pancreatic cancer. Br J Surg 2019;106:1590-601.

2. Kizy S, Altman AM, Wirth KM, et al. Systemic therapy 
without radiation may be appropriate as neoadjuvant therapy for localized pancreas cancer. Hepatobiliary Surg Nutr 2020;9:296-303.

3. Kirkegård J, Aahlin EK, Al-Saiddi M, et al. Multicentre study of multidisciplinary team assessment of pancreatic cancer resectability and treatment allocation. Br J Surg 2019;106:756-64.

4. Maxwell JE, Katz MHG. Radiotherapy for Resectable and Borderline Resectable Pancreas Cancer: When and Why? J Gastrointest Surg 2021;25:843-8.

5. Reni M, Balzano G, Zanon S, et al. Safety and efficacy of preoperative or postoperative chemotherapy for resectable pancreatic adenocarcinoma (PACT-15): a randomised, open-label, phase 2-3 trial. Lancet Gastroenterol Hepatol 2018;3:413-23.

6. Motoi F, Kosuge T, Ueno H, et al. Randomized phase II/ III trial of neoadjuvant chemotherapy with gemcitabine and S-1 versus upfront surgery for resectable pancreatic cancer (Prep-02/JSAP05). Jpn J Clin Oncol 2019;49:190-4.

Cite this article as: Søreide K. Heterogeneity in neoadjuvant therapy for localized pancreatic cancer: a persisting radiant autonomy. HepatoBiliary Surg Nutr 2021;10(2):254-256. doi: 10.21037/hbsn-2020-9
7. Conroy T, Desseigne F, Ychou M, et al. FOLFIRINOX versus gemcitabine for metastatic pancreatic cancer. $\mathrm{N}$ Engl J Med 2011;364:1817-25.

8. Conroy T, Hammel P, Hebbar M, et al. FOLFIRINOX or Gemcitabine as Adjuvant Therapy for Pancreatic Cancer. N Engl J Med 2018;379:2395-406.

9. Versteijne E, Suker M, Groothuis K, et al. Preoperative Chemoradiotherapy Versus Immediate Surgery for Resectable and Borderline Resectable Pancreatic Cancer: Results of the Dutch Randomized Phase III PREOPANC Trial. J Clin Oncol 2020;38:1763-73.

10. Jang JY, Han Y, Lee H, et al. Oncological Benefits of Neoadjuvant Chemoradiation With Gemcitabine Versus Upfront Surgery in Patients With Borderline Resectable Pancreatic Cancer: A Prospective, Randomized, Open-label, Multicenter Phase 2/3 Trial. Ann Surg 2018;268:215-22.

11. Del Chiaro M, Søreide K. Trials and tribulations of neoadjuvant therapy in pancreatic cancer. Br J Surg 2018;105:1387-9. 